\title{
Antiinflammatory effects of aprepitant coadministration with cART regimen containing ritonavir in HIV-infected adults
}

\author{
Sergei Spitsin, ${ }^{1}$ Pablo Tebas, ${ }^{2}$ Jeffrey S. Barrett, ${ }^{2}$ Vasiliki Pappa, ${ }^{1}$ Deborah Kim, ${ }^{2}$ Deanne Taylor, ${ }^{3}$ \\ Dwight L. Evans, ${ }^{4}$ and Steven D. Douglas ${ }^{1,2}$ \\ 'Department of Pediatrics, Children's Hospital of Philadelphia Research Institute, Philadelphia, Pennsylvania, USA. \\ ${ }^{2}$ Perelman School of Medicine, University of Pennsylvania, Philadelphia, Pennsylvania, USA. ${ }^{3}$ Department of Biomedical \\ and Health Informatics, Children's Hospital of Philadelphia Research Institute, Philadelphia, Pennsylvania, USA. \\ ${ }^{4}$ Department of Psychiatry, Perelman School of Medicine, University of Pennsylvania, Philadelphia, Pennsylvania, USA.
}

BACKGROUND. HIV-infected individuals, even well controlled with combined antiretroviral therapy (cART), have systemic inflammation and comorbidities. Substance $P(S P)$ is an undecapeptide, which mediates neurotransmission and inflammation through its cognate neurokinin 1 receptor (NI1R). Plasma SP levels are elevated in HIV-infected individuals. The FDA-approved antiemetic aprepitant, an NK1R antagonist, has anti-HIV effects and antiinflammatory actions. We evaluated the safety, pharmacokinetics, and antiinflammatory properties of aprepitant in HIV-positive individuals receiving CART.

METHODS. We conducted a phase 1B study of 12 HIV-positive individuals on a ritonavir-containing regimen (HIV viral load less than $\mathbf{4 0}$ copies $/ \mathrm{ml}$ and CD4 $>\mathbf{4 0 0}$ cells/ $\mu \mathrm{l}$ ). Participants received openlabel aprepitant $375 \mathrm{mg}$ per day for $\mathbf{2 8}$ days and were followed for an additional 30 days. Changes in plasma levels of proinflammatory markers were assessed using flow cytometry, ELISA, luminex, and SOMAscan assays.

RESULTS. The mean peak aprepitant plasma concentration was $30.7 \pm 15.3 \mu \mathrm{g} / \mathrm{ml}$ at day 14 and 23.3 $\pm 12.3 \mu \mathrm{g} / \mathrm{ml}$ at day 28. Aprepitant treatment resulted in decreased plasma SP levels and affected 176 plasma proteins (56 after FDR) and several metabolic pathways, including inflammation and lipid metabolism. No change in soluble CD163 was observed. Aprepitant treatment was associated with a moderate increases in total and HDL cholesterol and affected select hematologic and metabolic markers, which returned to baseline levels $\mathbf{3 0}$ days after aprepitant treatment was stopped. There were 12 mild and 10 moderate adverse events (AE).

CONCLUSIONS. Aprepitant is safe and well tolerated. The antiinflammatory properties of aprepitant make it a possible adjunctive therapy for comorbid conditions associated with HIV infection.

TRIAL REGISTRATION. ClinicalTrials.gov (NCT02154360).

FUNDING. This research was funded by NIH U01 MH090325, P30 MH097488, and P01 MH105303.

Conflict of interest: All authors were supported by grants from NIH. SDD has a patent US $20020094524 \mathrm{~A} 1$ issued.

Submitted: June 26, 2017 Accepted: September 6, 2017 Published: October 5, 2017

\section{Reference information:} JCI Insight. 2017;2(19):e95893. https://doi.org/10.1172/jci. insight. 95893.

\section{Introduction}

Combined antiretroviral therapy (cART) regimens improve the clinical and virologic outcomes in individuals with HIV infection (1-3). In individuals with sustained suppression of viral replication, evidence of chronic inflammation and immune dysfunction persists $(2,4)$. Immune dysregulation is associated with increased morbidity and mortality as a result of increase in the incidence of cardiovascular events, metabolic disorders, and HIV-associated neurocognitive disorders (HAND) (5-8). These comorbidities affect quality of life, adherence to antiretroviral therapy, employment, and overall survival. The limitations of the current HIV treatment regimens to curtail this ongoing inflammation are major justifications for continued research in anti-HIV therapeutics of new drugs with new mechanisms of action that target residual chronic inflammation. 
Table 1. Demographic characteristics

\begin{tabular}{|c|c|}
\hline Number of participants & 12 \\
\hline \multicolumn{2}{|l|}{ Sex } \\
\hline Male & $10(83 \%)$ \\
\hline Female & $2(17 \%)$ \\
\hline \multicolumn{2}{|l|}{ Age (years) } \\
\hline Mean (SD) & $47.0(8.6)$ \\
\hline Median (min, max) & $47.8(31.1,66.1)$ \\
\hline \multicolumn{2}{|l|}{ Ethnicity } \\
\hline Nonhispanic & $11(91.7 \%)$ \\
\hline Hispanic & $1(8.3 \%)$ \\
\hline \multicolumn{2}{|l|}{ Race } \\
\hline European descent & $2(17 \%)$ \\
\hline Black & $10(83 \%)$ \\
\hline
\end{tabular}

Substance P (SP) and its preferred receptor, neurokinin-1 receptor (NK1R), are central mediators in the interaction between the immune system and the nervous system (9). Compelling data support the concept that NK1R and the tachykinin, SP, are important in the pathogenesis of HIV/AIDS (9-13). HIV-infected men and women have elevated levels of circulating SP (14, 15). SP enhances HIV replication in macrophages, whereas NK1R antagonists inhibit it (11, 16-20). The SP autocrine loop has a role in regulating cytokine and inflammatory responses $(9,11,13)$. The NK1R antagonist, aprepitant, has unique features, which make it an attractive candidate to treat residual inflammation in $\operatorname{HIV}(9,21,22)$. Aprepitant (Emend) is a nonpeptide FDA-approved antiemetic drug licensed for the prevention of chemotherapy-induced nausea and vomiting (CINV) and postoperative nausea and vomiting. Aprepitant acts by binding and blocking the NK1R. Aprepitant crosses the blood-brain barrier (23) and has immunostimulatory and antiinflammatory properties $(9,13,24)$. Furthermore, aprepitant and other NK1R antagonists were successfully used to reduce symptoms of depression and insomnia in humans (25-27). In preclinical studies, NK1R antagonists had significant results - namely, reducing anxiety and inflammation, as well as an excellent safety record (reviewed in ref. 28). Most side effects associated with aprepitant administration are mild to moderate and include tiredness, hiccups, heartburn, stomach pain, diarrhea, constipation, loss of appetite, headache, dizziness, mild skin rash, sleep problems, and decrease in hemoglobin levels in children (29).

The current study is a dose and time escalation of our previous trials of aprepitant in HIV-positive patients (ClinicalTrials.gov [NCT00428519 and NCT01300988], refs. 21, 22) and, in addition, includes potentially novel biomarker measurements. In our previous study (NCT01300988), 9 cART-naive participants received aprepitant once-daily at a dose of $375 \mathrm{mg}$ for 2 weeks. The mean peak aprepitant plasma concentration on day 14 was $7.6 \pm 3.1 \mu \mathrm{g} / \mathrm{ml}$. Our studies provided support for a clinical exposure target above the one recommended for CINV. The approved 125-mg dose of aprepitant for CINV indication achieves $90 \%-99 \%$ receptor occupancy in positron emission tomography (PET) studies $(23,30,31)$. While partial receptor occupancy was sufficient to see improvement in insomnia, it may not be sufficient for other indications (for review, see ref. 32). Nearly $100 \%$ occupancy was required in order to demonstrate the effect of NK1R antagonist, casopitant, in major depression (33). The therapeutic dose of aprepitant for targeting chronic inflammation has not been established. Our results and modelling studies suggest that doses up to $375 \mathrm{mg}$ daily in HIV-infected participants elicited subtherapeutic, based on plasma levels, exposure of aprepitant, although effective plasma concentrations are likely achievable via proper dose modulation and using exposure enhancers such as CYP 3 A4 inhibitors $(34,35)$. The purpose of this clinical trial was to determine the safety of a longer aprepitant treatment ( $375 \mathrm{mg}$ per day for 4 weeks), as well as coadministration with the CYP 3A4 inhibitor ritonavir, in order to boost aprepitant plasma concentrations in virologically suppressed patients receiving cART (viral load below 40 copies/ml). In addition, this study evaluated the effect of aprepitant on proinflammatory markers and lipid metabolism for which we observed changes in our previous studies $(21,22)$, including increase in LDL, HDL, and total cholesterol. We used

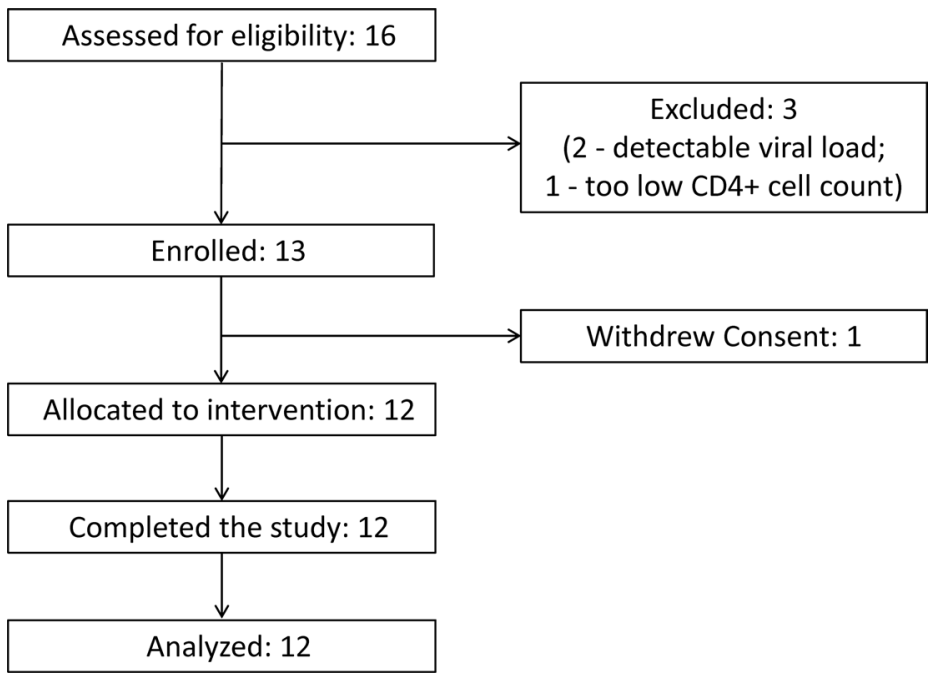




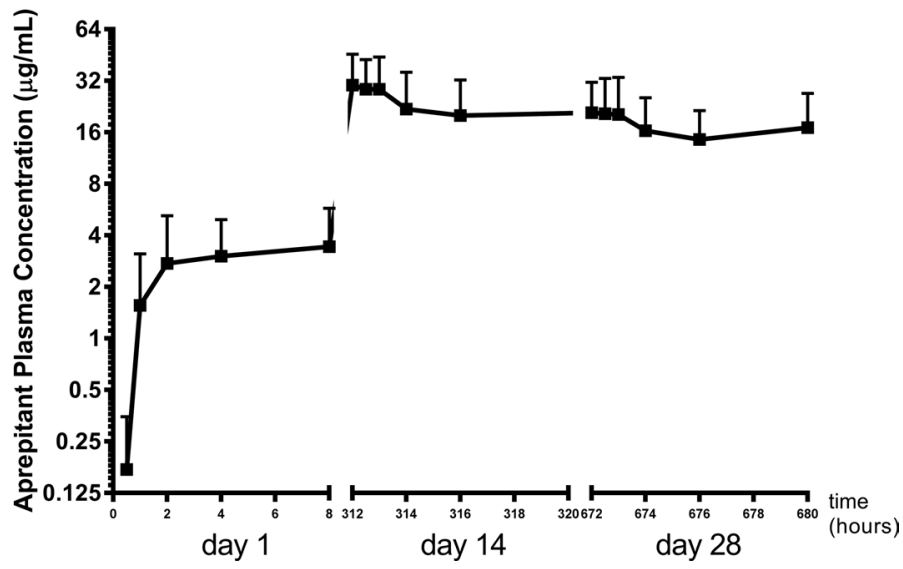

Figure 2. Plasma levels of aprepitant in patients receiving $375 \mathbf{~ m g}$ dose per day. Dose-exposure relationship. Eight-hour pharmacokinetic assessment was performed after the first dose (day 1 ) and at days 14 and 28 as indicated. In all cases, blood was drawn at $0.5,1,2,4$, and 8 hours after $375 \mathrm{mg}$ oral dose of aprepitant. Mean ( \pm SD) aprepitant plasma concentrations in 12 patients treated with drug are shown.

the proteomics platform, SOMAscan in order to assess effects of aprepitant on global protein expression in plasma. SOMAscan is a new proteomics discovery tool that utilizes modified single-stranded DNA-based reagents to examine the expression of 1,300 proteins in a multiplexed fashion $(36,37)$.

\section{Results}

Twelve participants were enrolled and completed the study. Table 1 and Figure 1 summarize the demographic characteristics of the participants.

The mean peak aprepitant plasma concentrations were $4.1 \pm 2.7 \mu \mathrm{g} / \mathrm{ml}$ on day $1,30.7 \pm 15.3 \mu \mathrm{g} / \mathrm{ml}$ at day 14 , and $23.3 \pm 12.3 \mu \mathrm{g} / \mathrm{ml}$ at day 28 , as shown in Figure 2 . The time of peak plasma concentration (Tmax) was $5.4 \pm 3.2$ hours on day $1,0.8 \pm 0.3$ hours on day 14 , and $2.2 \pm 2.8$ hours on day 28 .

None of the patients were clinically depressed at enrollment or during the study. Ten of 12 patients did not have significant anxiety or sleep-quality issues. Two patients (patient IDs [PIDs] 001 and 011) had a Hamilton Anxiety score of 8 and 20, respectively, and the highest sleep disturbance score ( 9 and 10, respectively) at enrollment. Aprepitant treatment was associated with a transient decrease in both (Hamilton Anxiety scores at day 28 : 4 and 2, respectively; Pittsburgh Sleep Quality Index: 6 and 4, respectively). Similar observations were recorded in Hamilton Depression Rating Scale (17 item) for PID 001: decrease from 7 to 2 during aprepitant treatment.

Table 2. Adverse events by preferred term and subject, $n$ (\% of total individuals with adverse event)

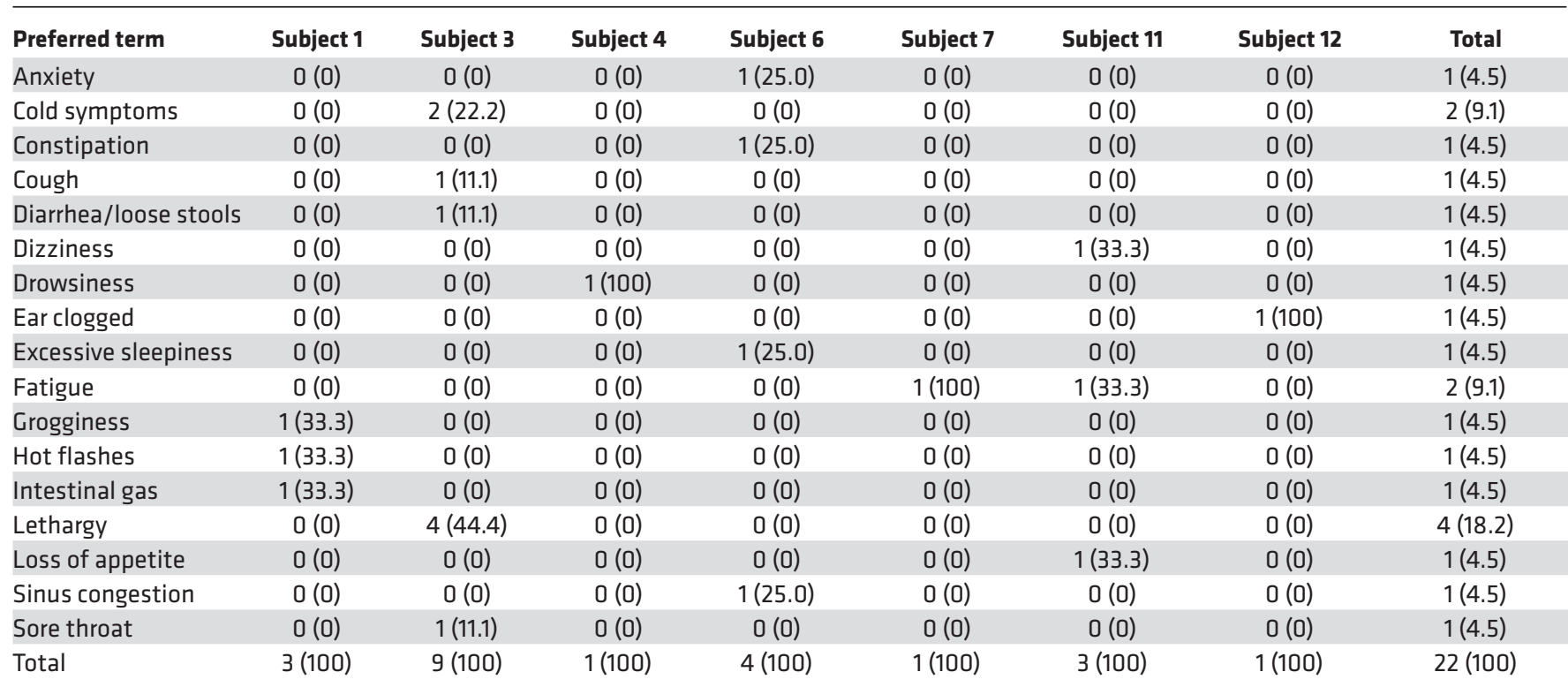



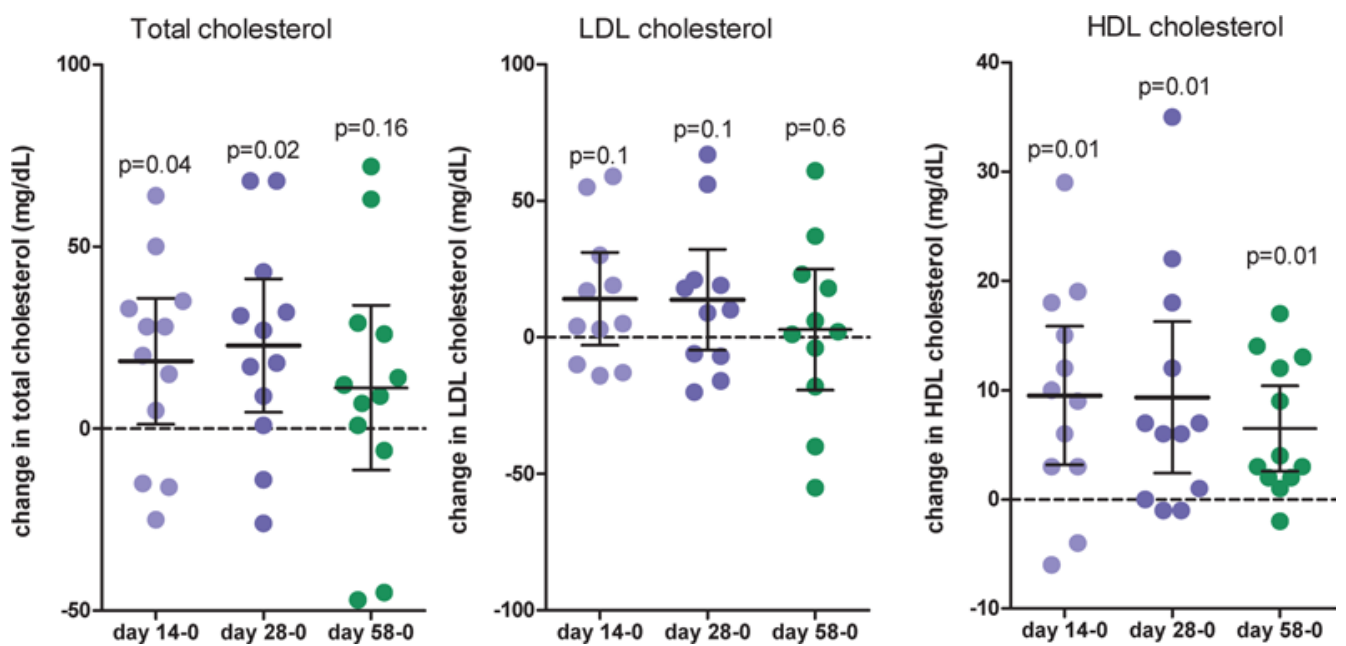

Figure 3. Changes in cholesterol levels associated with aprepitant treatment. Total cholesterol, LDL, and HDL were measured by Quest Diagnostics. Results are presented as changes in cholesterol levels between day 0 and 14 , day 0 and 28 , or day 0 and 58 as indicated. Vertical bars are the widths of the $95 \% \mathrm{CI}$. $P$ values by Wilcoxon signed-rank test are indicated.

None of the changes were significant for the entire group of 12 patients (Supplemental Figure 1; supplemental material available online with this article; https://doi.org/10.1172/jci.insight.95893DS1).

There were no serious adverse events (AE) associated with 4 weeks of treatment with $375 \mathrm{mg}$ of aprepitant. AE were mild to moderate and self-limited in nature. Five patients did not have AE (Table 2 and Supplemental Table 1).

We observed a decrease in plasma hemoglobin levels at day 28 of treatment with aprepitant (mean decrease $-0.61 \pm 0.56 \mathrm{~g} / \mathrm{dl}, P=0.01$ ). The overall hemoglobin decrease was less than $1 \mathrm{~g} / \mathrm{dl}$. In one patient (PID 001), hemoglobin levels dropped below $10 \mathrm{~g} / \mathrm{dl}$, to $9.9 \mathrm{~g} / \mathrm{dl}$ (Supplemental Figure 2A). Similar to hemoglobin, hematocrit and $\mathrm{rbc}$ were decreased at day 28 (hematocrit mean decrease $-1.5 \% \pm 1.78 \%, P=$ 0.03 ; rbc mean decrease $-0.19 \times 10^{6} \mathrm{cells} / \mu 1 \pm 0.21 \times 10^{6} \mathrm{cells} / \mu 1, P=0.02$ (Supplemental Figure 2, B and C). Both hemoglobin and rbc returned to baseline by day 58 (30 days after treatment was discontinued).

Aprepitant treatment was associated with minimal increase in platelets $\left(9.92 \pm 16.17 \times 10^{3} / \mu 1, P=\right.$ 0.02 ) at day 28 (Supplemental Figure 3). The use of aprepitant was associated with increases in plasma lipid levels (Figure 3). The mean change in total cholesterol within the subject at the end of treatment (day $28)$ was $+23 \mathrm{mg} / \mathrm{dl}(P=0.02)$. The mean change in LDL cholesterol was $+14 \mathrm{mg} / \mathrm{dl}(P=0.1)$ and mean change in HDL was $+9 \mathrm{mg} / \mathrm{dl}(P=0.01)$. These lipid levels returned to baseline values by day 58 , after treatment was discontinued, with the exception of HDL. HDL levels remained increased 30 days after aprepitant treatment was stopped. No significant changes were observed in triglyceride levels.

In order to evaluate the effect of SP-NK1R pathway on cholesterol uptake by human macrophages, we incubated human monocyte-derived macrophages (MDMs) with acetylated cholesterol spiked with ${ }^{3} \mathrm{H}$-cholesterol. Incubation of MDM with acetylated cholesterol resulted in formation of foam cells detected by Oil Red O staining (Figure 4A). SP treatment increased cholesterol uptake by human macrophages in vitro, and aprepitant blocked this effect (Figure 4B).

The metabolic panel studies demonstrated decreases in total bilirubin and increases in ALT, which were not clinically significant (Supplemental Figure 4).

We observed a decrease in absolute counts of $\mathrm{CD}^{+} \mathrm{T}$ cells (but not in percentage) at day 14 of aprepitant treatment. The decrease was generally small $(-91.4 \pm 131.8,-78.5 \pm 119.2$, and $-50.1 \pm$ 143.6 cells $/ \mathrm{ml}$, mean \pm SD, on days 14,28 , and 58 respectively; Supplemental Figure 5). The CD4 ${ }^{+}$ count remained above 350 in all patients and was not associated with an increase in HIV viral loads. All patients had viral loads below detectable levels before and after aprepitant treatment, as measured by Amplicor HIV-1 Monitor v1.5 (Roche Diagnostics), with a sensitivity of 40 copies $/ \mathrm{ml}$. A high sensitivity viral load assay performed before and after aprepitant treatment (28 days) by the HIV Molecular Monitoring Core at the Frederick National Laboratory for Cancer Research (AIDS and Cancer Virus Program, Leidos Biomedical Research Inc., Frederick, Maryland, USA) identified 4 patients 
A

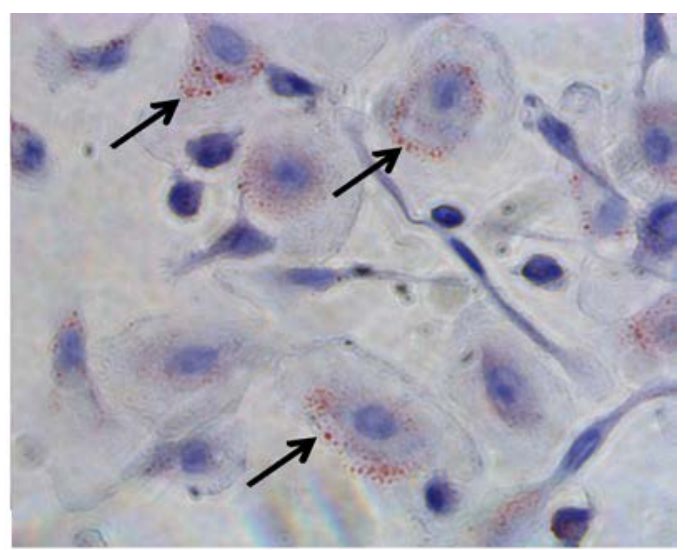

B

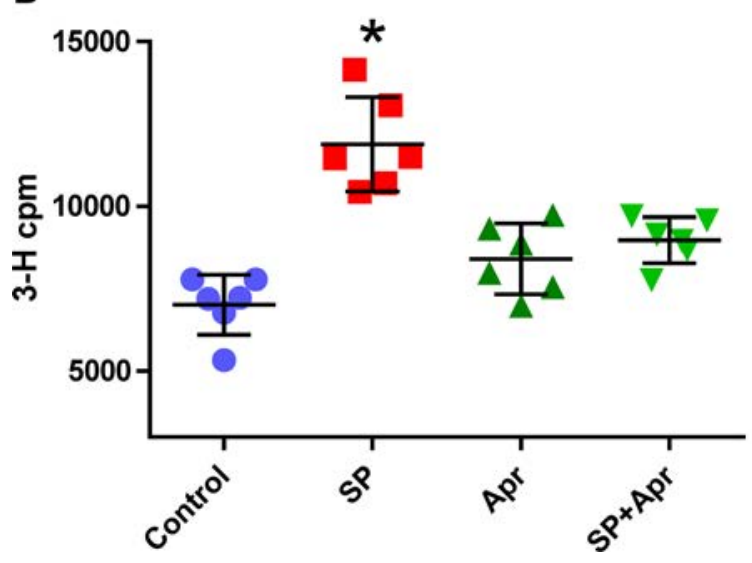

Figure 4. Effect of substance $P(S P)$ and aprepitant on lipid accumulation by human monocyte derived macrophages (MDM). (A) Lipid accumulation by human macrophages. Human MDM were differentiated for 7 days in vitro as described in Methods. After incubation with acetylated LDL from human serum for 24 hours, cells were stained with Oil Red 0 stain. Lipid inclusions (red) are shown with arrows. Magnification, $\times 400$. (B) Quantification of lipid accumulation by human MDM. Cholesterol $\left[1,2^{-3} \mathrm{H}(\mathrm{N})\right](1 \mu \mathrm{Ci})$ was mixed with $50 \mu$ g of purified acetylated $\mathrm{LDL}$ from human serum, incubated for 30 minutes, and then added to MDM cultures and incubated for additional 24 hours. SP and aprepitant (Apr), $10 \mu \mathrm{M}$ each, were added as indicated. Cells were washed with PBS and lysed with $10 \%$ Tween $20 .{ }^{3} \mathrm{H}$ was measured in cell lysates using $\beta$-counter. The results are presented as individual values from 3 independent experiments, each done in duplicates. Vertical bars are mean \pm $\mathrm{SD}$. Each experiment is done with cells from different donors. Each condition is done in duplicates. ${ }^{*} P<0.05$ by Student's $t$ test for SP-treated samples vs. not-treated control, vs. Apr, or vs. SP+Apr.

with viral loads between 1 and 14 copies/ml. Seven patients had a viral load below 1 copy $/ \mathrm{ml}$. The results for one patient were not measurable due to sample DNA contamination (Supplemental Table 2).

We observed a decrease in plasma SP levels associated with aprepitant treatment (Figure 5). This observation is consistent with our previous reports $(21,22)$. No significant changes in PD-1 expression in T cells or soluble CD163 (sCD163) levels in the plasma of patients undergoing aprepitant treatment were detected. We observed an increase in central memory $\mathrm{CD} 4^{+}$and $\mathrm{CD} 8^{+}$ $\mathrm{T}$ cells $(\mathrm{Tcm})$ associated with aprepitant treatment (Supplemental Figure 6) and a return to baseline at day 58, 30 days after aprepitant was discontinued.

The SOMAscan assay identified 176 proteins ( 56 after FDR adjustment) that were affected by aprepitant administration and associated with several metabolic pathways, including inflammation apoptosis and lipid metabolism pathways (Figure 6). Changes in several individual proteins representing different pathways are shown in Figure 7. A complete list of the plasma proteins altered by aprepitant treatment are presented in Supplemental Table 3.

\section{Discussion}

HIV-positive patients on stable cART and controlled viral replication received $375 \mathrm{mg}$ of aprepitant daily. The treatment with aprepitant was safe and well tolerated over the 4 weeks of drug administration. The administration of aprepitant in combination with CYP 3A4 inhibitor ritonavir resulted in a significant boost in plasma levels of drug. The mean peak aprepitant plasma concentrations on day 14 was $30.7 \pm 15.3 \mu \mathrm{g} / \mathrm{ml}$ in the current study vs. $7.6 \pm 3.1 \mu \mathrm{g} / \mathrm{ml}$ when administered as monotherapy (22). This study did not have a placebo group; $22 \mathrm{AE}$ were reported (12 mild, 10 moderate, and 0 severe) in the 12 individuals. This is comparable with the observed $\mathrm{AE}$ reported for the placebo group in our previous study: 11 mild and 11 moderate in 9 HIV-positive patients receiving placebo for 2 weeks (22). A major pharmacologic outcome of this trial was that we did not observe the reduction in aprepitant exposure at 4 weeks due to enzyme induction; that was previously observed preclinically in various animal species (38) and in humans in earlier depression trials (39). As previously discussed (34), this outcome is likely attributed to the competition for the CYP3A4 enzyme due to inhibition with ritonavir and other $3 \mathrm{~A} 4 \mathrm{sub}-$ strates in this population. Moreover, the higher exposures observed

Figure 5. Changes in substance $\mathbf{P}$ (SP) levels associated with aprepitant treatment. SP levels were determined as described in the Methods section. The results are presented as changes in SP levels between day 0 and 14 , day 0 and 28 , or day 0 and 58 as indicated. Vertical bars are the widths of the $95 \% \mathrm{Cl}$. $P$ values determined using Wilcoxon signed-rank test are indicated.

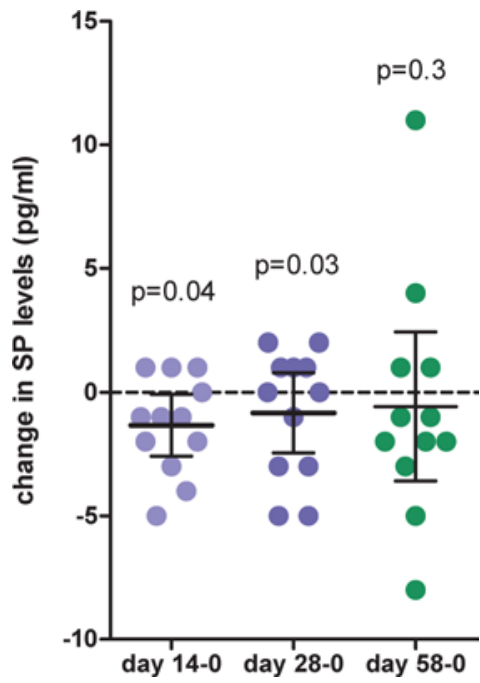




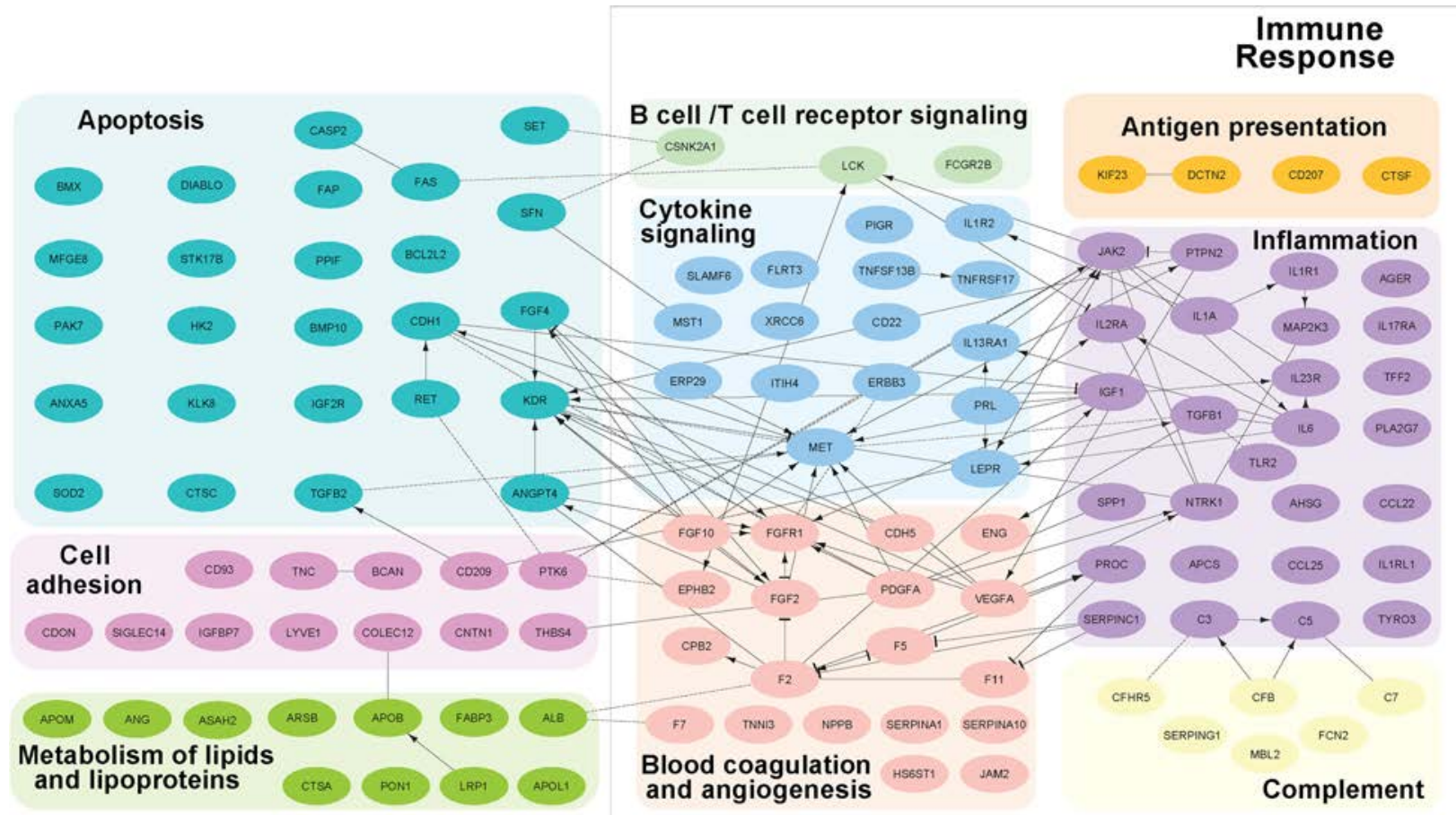

Figure 6. Protein interaction network for proteins that were affected by aprepitant administration. Linear mixed effect modeling identified 176 proteins that changed their expression from baseline to day 28 after aprepitant treatment. The Reactome FIViz plugin of the Cytoscape software was used to identify metabolic pathways and plot a protein interaction network. Linker genes were included in the analysis. Linker genes and proteins that were not members of pathways in one of the 9 categories are hidden from view. $(\rightarrow)$ denotes activating or catalyzing functional interactions, $(-\mid)$ denotes inhibiting interactions, $(-)$ denotes proteins that are part of a complex or input, and (- - -) denotes predicted protein interactions.

in this trial were not associated with any unanticipated safety findings, suggesting that higher doses can be attained in order to reach the desired clinical indices. We did not observe any AE leading to study termination or indicating any immediate warning associated with prolonged use of aprepitant.

The administration of aprepitant was not associated with changes in SCD163. We confirmed our previous observations that aprepitant treatment is associated with decreases in plasma levels of SP $(21,22)$. In our previous study of $375 \mathrm{mg}$ aprepitant daily for 2 weeks, we observed a treatment-associated decrease in markers of inflammation/immune exhaustion including SCD163 and PD-1 (22). However, we did not observe this effect in the current 4-week treatment study. The SOMAscan assay demonstrated that aprepitant treatment was associated with a decrease in plasma levels of the immune checkpoint marker, lymphocyte-activation gene-3 (LAG3). The differences between the current and previous studies may be related to higher initial baseline levels of proinflammatory markers in cART-naive patients and the small sample size of this study. The initial baseline of sCD163 in the HIV viremic population was in the 1,500-4,000 ng/ml range (22), while in the current study, patients with undetectable viral loads had SCD163 levels, which were in the 400-1,800 ng/ml range.

Minor decreases in hemoglobin, hematocrit, and $\mathrm{rbc}$ are likely related to aprepitant administration. The decrease in hemoglobin in children was previously reported by the manufacturer as a side effect of aprepitant administration (29). This may be a result of SP effects on cell proliferation, including CD34 in the BM (40). The effect is blocked by aprepitant (our unpublished observations). Although not a major clinical concern in the current study, hemoglobin levels should be monitored in future studies involving prolonged administration of aprepitant or other NK1R antagonists.

The observed increase in platelet number associated with aprepitant treatment is unlikely to have any biological significance due to a very small absolute increase (less than 10,000 platelets/ $\mu$ ), with platelets remaining within normal levels for all individuals. The changes in platelets are likely to be related to aprepitant treatment, since data of SOMAscan analysis show changes in a number of proteins related to blood coagulation and angiogenesis pathways (Figure 6 and Supplemental Table 3).

We observed increases in plasma cholesterol associated with the use of aprepitant that were consistent with our previous study (22). The magnitude of the cholesterol level increase observed in the current study 

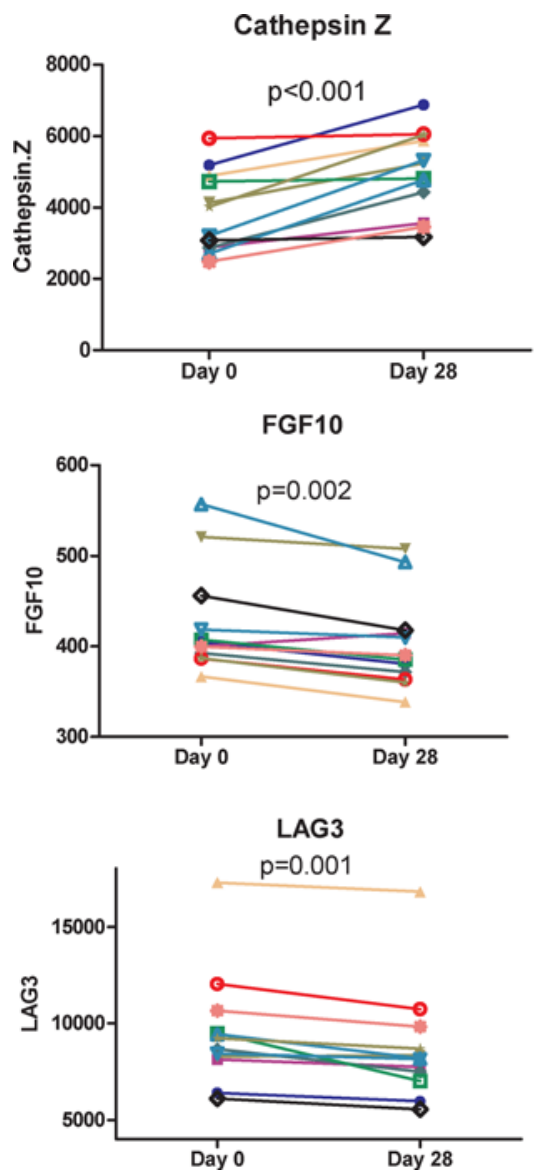

\section{IL-1 receptor like 1}
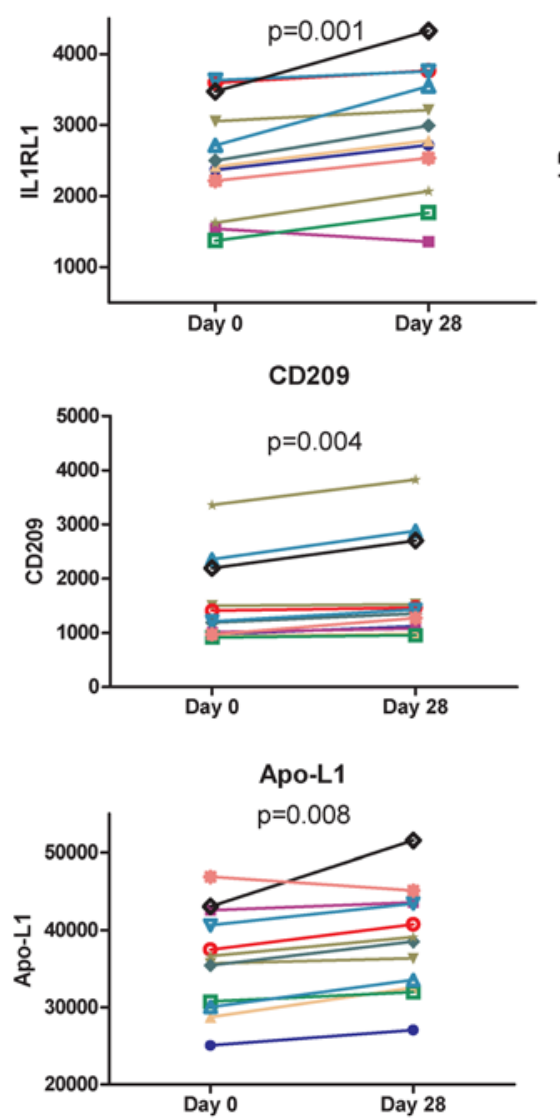

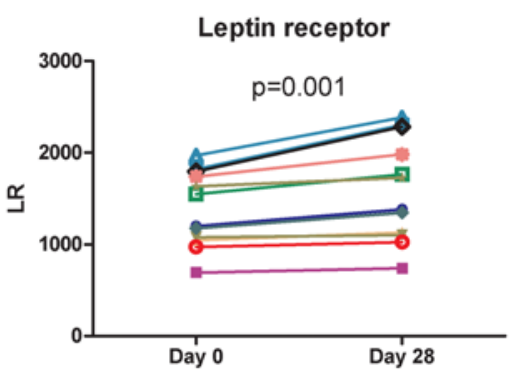

$\rightarrow$ PID 1

- PID 2

- PID 3

-PID 4

$\rightarrow$ PID 5

- - PID 6

\# PID 7

¿ PID 8

$\rightarrow$ PID 9

๑ PID $1 C$

- - PID 11

Alpha-1-antitrypsin $\quad \div$ PID 12

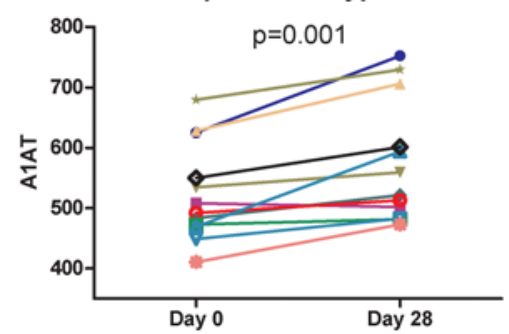

IL-6

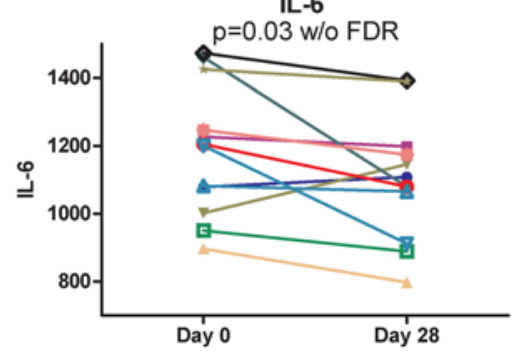

Figure 7. Changes in selected individual proteins affected by aprepitant treatment. Changes in plasma levels of individual proteins as a result of aprepitant treatment are shown. $P$ values determined by 2-way ANOVA-FDR with the exception of IL-6, where $P$ value is shown without the FDR adjustment. Changes in IL-6 levels are consistent with our previous studies (22).

was not greater than that observed in previous studies, despite an increase in study duration and the higher aprepitant plasma levels achieved. The overall increase was relatively small, with a more profound increase in "good" HDL cholesterol. There are no available data on the direct effect of either SP or aprepitant on cholesterol metabolism. Patients with advanced HIV infection have low HDL cholesterol levels and other lipid abnormalities, including hypertriglyceridemia and increased levels of small LDL particles (reviewed in ref. 41), which may be directly or indirectly related to the increased circulating levels of SP in HIV-infected individuals $(14,15)$. SP has been demonstrated to affect lipid metabolism. Increased SP levels occur with obesity and diabetes in human SP and in mouse models (for review, see ref. 42). NK1R-KO mice are more resistant to the development of obesity (43); similar effects were observed using NK1R antagonists (44). The effect of aprepitant on lipid/cholesterol metabolism was further confirmed by the SOMAscan assay, which identified changes in a number of proteins related to cholesterol and lipid metabolism (Figures 6 and 7, and Supplemental Table 3), including an increase in the plasma levels of Apo-L1 and soluble leptin receptor, which may facilitate weight loss by blocking leptin (45). SP treatment increases cholesterol absorption by human macrophages and formation of foam cells, whereas aprepitant blocks this effect. The increase in circulating cholesterol levels observed in vivo in individuals treated with aprepitant is possibly due to decrease of cholesterol absorption in macrophages. Although increased plasma cholesterol occurs with the development of atherosclerosis, decreased cholesterol accumulation in blood vessel macrophages and an increase of "good" HDL cholesterol may have an overall ameliorating effect on atherogenesis.

The significance of observed changes in bilirubin and liver enzymes are uncertain. Aprepitant treatment is associated with a low rate of serum enzyme elevations during therapy but has not been linked to cases of clinically apparent liver injury (46). Aprepitant is metabolized in the liver by CYP3A4, with minor metabolism performed by CYP1A2- and CYP2C19-dependent mechanisms (29). These changes persisted after 
completion of aprepitant treatment. We did not observe similar changes in the 2-week-long treatment (22). Longer administration and/or coadministration with cART - which includes atazanavir, known to affect liver function - may be responsible for changes in liver panel and should be monitored in any future studies.

The observed increase in both $\mathrm{CD}^{+}$and $\mathrm{CD} 8^{+} \mathrm{Tcm}$ is not explained by changes in viral load but may be explained by aprepitant's inhibition of the SP-NK1R signaling pathway, which may alter lymphocyte trafficking and homing through regulation of chemokines and adhesion molecules (47-50). This hypothesis is supported by the observation that we did not detect changes in $\mathrm{CD} 4^{+}$percentages.

A decrease, although modest, in the $\mathrm{CD}^{+} \mathrm{T}$ cells during aprepitant treatment was an unexpected observation. The maximum decrease occurred 2 weeks after the start of drug administration. We did not observe a similar effect in previous studies in cART-naive patients, although we used a similar dose of 375 $\mathrm{mg}$ of aprepitant daily. The administration of aprepitant, in combination with CYP 3A4 inhibitor ritonavir, led to 3- to 4-fold higher plasma levels of the aprepitant. This effect is not mediated by HIV rebound, since no increase in viral RNA was observed as measured either by conventional or a high-sensitivity HIV viral assay capable of detecting a single copy of viral RNA. The possible explanations for aprepitant-mediated decrease in $\mathrm{CD}^{+} \mathrm{T}$ cells may be related to aprepitant inhibition of the SP ability to increase cell proliferation and/or to decrease cell apoptosis $(9,51)$.

The analysis of SOMAscan data showed changes in several pathways related to the immune response, including inflammation, cytokine, and lymphocyte signaling and complement, suggesting a significant effect of aprepitant on overall immune regulation. A number of changes detected by SOMAscan are consistent with observations of the proinflammatory role of SP $(9,52,53)$ and our hypothesis of the antiinflammatory effect of aprepitant. We detected a decrease in IL-6 levels - an important cytokine in chronic, HIV-associated inflammation - although to a lesser extent than in the previous study. Perhaps this was due to the small number of patients studied and the relatively low background inflammation in these subjects. HIV replication inhibits CD209+ expression by human macrophages, driving them toward a proinflammatory phenotype (54). The observed increase in $\mathrm{SCD}^{2} 09^{+}$, a mannose receptor, with aprepitant treatment (Figure 7) supports our hypothesis that NK1R antagonism has antiinflammatory effects. To our knowledge, there is no direct relationship known between FGF10 expression and HIV infection. Decrease in FGF10 (Figure 7), a cytokine that increases cell proliferation and cell survival, may reflect aprepitant inhibition of SP/NK1R signaling, also known to promote cell proliferation and apoptosis. Alterations in FGF10 levels have been reported in physiological and pathophysiological conditions, which include tissue development, repair, growth, and progression (55). In addition to previously reported markers of inflammation/immune dysregulation, the SOMAscan assay identified a number of potential new targets affected by aprepitant treatment (Figure 6 and Supplemental Table 3). Some of these markers, although associated with inflammation, may have protective functions, (e.g., increases in IL-1RL1 have been linked to cardio protection, and $\alpha 1$-antitrypsin, a protein with potent protease inhibitor activity, is known to protect normal tissues from proteolytic attack during inflammation). Cathepsin $\mathrm{Z}$, although known to be implicated in inflammation, may also have immune-protective properties (56). The relevance of these markers to chronic HIV infection and the antiinflammatory potential of aprepitant requires additional investigation.

The prolonged exposure to NK1R antagonist aprepitant has a wide spectrum of effects on human physiology, including cholesterol/lipid metabolism. Although these changes did not have direct clinical relevance, further studies using long-term aprepitant and other NK1R antagonists require metabolic monitoring. Our results confirm and extend the observation of the SP/NK1R signaling effect on inflammation, cell trafficking, and apoptosis. We have identified new targets linked to these pathways, justifying further studies of aprepitant in patients with cART-treated HIV and incompletely suppressed inflammation. The inhibition of cholesterol uptake of macrophages by blocking the NK1R receptor has implications in the prevention of atherosclerosis and atherosclerotic plaque evolution and merits further evaluation.

\section{Methods}

\section{Study design and study procedures}

A phase $1 \mathrm{~B}$ open label study to evaluate the safety and pharmacokinetics of the NK1R antagonist aprepitant in HIV-infected adults receiving cART, containing ritonavir with plasma HIV viral load less than 40 copies $/ \mathrm{ml}$ and CD4 $>350$ cells/ $\mu 1$, was conducted. Sixteen participants with HIV-1 infection, 
on stable antiretroviral therapy, gave consent for the trial. One participant withdrew consent, 2 participants had detectable viral loads (greater than 40 copies $/ \mathrm{ml}$ ), and one participant had CD4 ${ }^{+}$counts below 350 cells $/ \mu 1$. Twelve patients were enrolled in the study and received aprepitant $375 \mathrm{mg}$ once a day for 28 days, and they were followed for an additional 30 days off the study drug. Six patients were on atazanavir $300 \mathrm{mg} /$ ritonavir $100 \mathrm{mg}$ daily and 6 were on a darunavir $800 \mathrm{mg} /$ ritonavir 100 mg daily antiretroviral-containing regimen. The 12 patients enrolled completed the 58 days follow-up visit. Safety laboratory tests and HIV-1 RNA (Amplicor HIV-1 Monitor v1.5, Roche Diagnostics) were conducted at days $0,3,7,10,14$, and 28 while participants were receiving aprepitant, and at day 58 (4 weeks after discontinuing study medication). Safety tests were conducted as part of this Phase $1 \mathrm{~B}$ study. Additionally, an 8-hour pharmacokinetic assessment was performed after the first dose and at days 14 and 28. The Hamilton-17 Depression Rating Scale (HAM-D-17), the Hamilton Anxiety Scale (HAM-A), and Pittsburgh Sleep Quality Index (PSQI) total scores were assessed on days 0, 28, and 58.

Study objectives. Primary endpoints: (i) to assess the safety and tolerability of aprepitant when administered for 4 weeks in combination with a ritonavir-containing antiretroviral regimen; (ii) to assess the pharmacokinetic characteristics of aprepitant when coadministered with a ritonavir-containing antiretroviral regimen; and (iii) to evaluate the effects of aprepitant on plasma levels of SP and SCD163 in patients with well-controlled viral replication. Secondary objectives: (i) to evaluate the effects of aprepitant on levels of CD4/PD1 expression in patients with well-controlled viral replication; (ii) to evaluate the effects of aprepitant on lipid metabolism; and (iii) to evaluate the effects of aprepitant on circulating proinflammatory cytokines and chemokines.

\section{Participants}

Participants were HIV-infected individuals, older than 18 years of age, receiving antiretroviral treatment with a regimen that includes either atazanavir/ritonavir or darunavir/ritonavir for at least 6 months prior to enrollment, with $\mathrm{CD}^{+}$cell counts greater than $350 \mathrm{cells} / \mathrm{mm}^{3}$ and plasma HIV-1 RNA below the limit of quantification as measured by the standard ultrasensitive assay (e.g., Roche Amplicor Monitor v1.5 assay). We excluded individuals with a history of cancer, serious illness, pregnancy, chronic hepatitis B or C infection; individuals with significant laboratory abnormalities; individuals using steroids or any other immunomodulators or chemotherapy; and individuals with allergy or hypersensitivity to aprepitant. We also excluded individuals using inhibitors or inducers of cytochrome P450 3A4 metabolism, with the exception of the protease inhibitors administered in this trial.

\section{Bioanalysis and pharmacokinetics}

A validated, liquid chromatography-tandem mass spectrometry method was used for the quantification of aprepitant plasma concentration in HIV-infected individuals and tissue culture media. The method utilized a simple sample-preparation procedure of protein precipitation with methanol. Chromatographic separation was performed on a reversed phase C8 column (Hypersil Gold, $50 \mathrm{~mm} \times 2.1 \mathrm{~mm}$, $3 \mu \mathrm{m}$ ) using a mobile phase composed of acetonitrile and water in $0.5 \%$ formic acid through gradient elution (Thermo Fisher Scientific). Electro-spray ionization in positive mode was incorporated in the tandem mass spectrometric detection. The lower limit of quantitation of aprepitant in human plasma was $1 \mathrm{ng} / \mathrm{ml}$. The details of the method validation have been previously published (57).

\section{Laboratory assays}

Multiplex SP and cytokine assays. Concentrations of IFN $\gamma$, IL-1 $\beta$, IL-6, IL-8, IL-10, and TNF $\alpha$ in human plasma were measured using the human custom-made Magnetic Bead Panel (MilliporeSigma). The concentration of SP was measured using the Neuropeptide Kit HNPMAG-35K (MilliporeSigma) according to manufacturer's recommendations.

sCD163 assay. sCD163 was measured an ELISA assay kit (Trillium Diagnostics) according to manufacturer's recommendations.

Flow cytometry. Blood samples were collected in anticoagulant (EDTA) and stained within 24 hours with monoclonal antibodies (including anti-CD4 and anti-PD-1) conjugated with fluorescent labels purchased from Biolegend, BD Biosciences, and Trillium Diagnostics (Supplemental Material). After erythrocytes lysis, cells were fixed and analyzed on an LSR II flow cytometer (BD Biosciences). Compensation for fluorescence spillover and data analysis was performed using the FACSDiva software (BD Biosciences). 
High-sensitivity viral load assay. High-sensitivity viral load assay was performed by the HIV Molecular Monitoring Core at the Frederick National Laboratory for Cancer Research (AIDS and Cancer Virus Program).

SOMAscan assay. SOMAscan assay on plasma samples at day 0 and day 28 was performed by the manufacturer (SomaLogic). SOMAscan assay targets 1,300 proteins that represent a diverse set of pathways and molecular functions, including cancer, inflammation, and cardiovascular conditions. Targets include receptors, kinases, growth factors, and hormones, as well as secreted intracellular and extracellular proteins and domains.

In vitro cholesterol uptake by human MDMs and formation of foam cells. Deidentified, fresh monocytes (purity $>$ 95\%) from HIV-negative donors were purchased from the Human Immunology Core Facility of the University of Pennsylvania School of Medicine (Philadelphia, Pennsylvania, USA). The monocytes were purified from apheresis material by negative selection using RossetteSep kit (Stem Cell Technologies). Unwanted cells were targeted for removal with Tetrameric Antibody Complexes recognizing CD2, CD3, CD8, CD19, CD56, CD66b, CD123, and glycophorin A. The monocytes were washed in DMEM and cultured in 24-well plates; 250,000 cells per well in DMEM were supplemented with $10 \% \mathrm{FBS}$ and antibiotics at $37^{\circ} \mathrm{C}$ in $5 \%$ $\mathrm{CO}_{2}$ for 7 days to generate MDM. For cholesterol uptake experiments, MDM were incubated with $50 \mu \mathrm{g} /$ $\mathrm{ml}$ of purified acetylated LDL from human serum (AbD Serotec, Bio-Rad) for 24 hours and stained with Oil Red O stain (MilliporeSigma). For the quantification of lipid accumulation by human MDM, $1 \mu \mathrm{Ci}$ of cholesterol $\left(1,2-{ }^{3} \mathrm{H}[\mathrm{N}]\right.$; PerkinElmer), was mixed with $50 \mu \mathrm{g}$ of purified acetylated LDL from human serum (AbD Serotec,Bio-Rad) in $50 \mu \mathrm{l}$ of DMEM, incubated for 30 minutes, and then added to MDM cultures and incubated for 24 hours. SP and aprepitant (10 $\mu \mathrm{M}$ of each) were added to some wells. Cells were washed with PBS and lysed with $10 \%$ Tween $20 .{ }^{3} \mathrm{H}$ was measured in cell lysates using a $\beta$-counter (Beckman Coulter).

\section{Statistics}

Descriptive statistical analyses of all data collected were conducted using inferential and graphical exploratory data analytic techniques. Descriptive statistics for continuous variables were summarized by computing the mean, standard deviation, median, minimum value, and maximum value. Changes from baseline to days 14,28 , and 58 were computed for each participant for each continuous variable. Categorical variables were summarized as counts and percentages of the total. The Wilcoxon signed-rank test was used to test whether the within-subject changes from baseline to days 14, 28, or 58 were significantly different than zero. The significance level for 2 -sided testing was set at $P=0.05$. Stata version 12.1 (StataCorp) was used for descriptive statistics, producing graphs and tables, and conducting the statistical tests.

SOMAscan data were analyzed with the lme4 package (58) in $\mathrm{R}$ (59). The data were fitted to a linear mixed effect model to detect significant changes in protein expression from baseline to day 28, with PID as the random effect variable. The car $\mathrm{R}$ package was used to calculate likelihood ratio tests and obtain $P$ values for the coefficients of the fixed effects (60). $P$ values and FDR adjusted values below 0.05 were considered statistically significant. Protein interaction networks, for proteins with $P$ values below 0.05 , were created with the Reactome FIViz application in the Cytoscape (61). Linker genes were included in the analysis. The results from both Pathway enrichment and Gene Ontology Biological Process analysis were used to create the 9 categories of the network.

\section{Study approval}

The study was conducted at the AIDS Clinical Trials Unit and the Clinical and Translational Research Center (CTRC) of the Hospital of the University of Pennsylvania in Philadelphia, Pennsylvania, USA. All patients signed a written informed consent. The study was sponsored by the National Institutes of Mental Health, approved by the IRB of the University of Pennsylvania and the US Food and Drug Administration (IND\#75,558), and registered in Clinical Trials.gov (NCT02154360).

\section{Author contributions}

SS, PT, DK, JSB, DLE, and SDD conceived and designed the experiments. PT, DK, SS, and SDD performed the experiments. SS, PT, JSB, VP, DT, and SDD analyzed the data. SS, PT, VP, DLE, and SDD wrote the paper.

\section{Acknowledgments}

This work was supported by the NIH grants NIH UO1 MH090325, P30 MH097488, and PO1 MH105303. We thank Robert J. Gorelick, Jeffrey Lifson, Frank Maldarelli, and Brandie Fullmer from Brandie of HIV Molecular Monitoring Core, AIDS and Cancer Virus Program, Frederick National Laboratory for Cancer Research, Leidos Biomedical Research, Inc., Frederick, Maryland, USA, for performing the high sensitiv- 
ity HIV viral load assay (see Supplemental Table 2); Ro Kappes and the staff from the Clinical Research Site of the ACTG of the Hospital of the University of Pennsylvania who graciously recruited and followed the participants for this study; Nancy Tustin, Richard Tustin III, and Laura A. Schankel, PMHARC, Laboratory and Biobehavioral Core E, for technical support; Ganesh Moorthy and Athena F. Zuppa, Bioanalytical Core Laboratories, Center for Clinical Pharmacology at Children's Hospital of Philadelphia for performing pharmacokinetics assessments; Florin Tuluc and the Flow Cytometry Core Laboratory at The Children's Hospital of Philadelphia for providing flow cytometry services; Okan Elci, Westat, Rockville, Maryland, USA, for statistical analysis; Zenobia Cofer for editorial assistance and the study participants for their commitment and contribution to this clinical trial.

Address correspondence to: Steven D. Douglas, Children's Hospital of Philadelphia, 34th Street and Civic Center Blvd., Abramson Building, Room 1208A, Philadelphia, Pennsylvania 19104, USA. Phone: 215.590.3561; Email: douglas@email.chop.edu.

1. Eisele E, Siliciano RF. Redefining the viral reservoirs that prevent HIV-1 eradication. Immunity. 2012;37(3):377-388

2. Chun TW, Fauci AS. HIV reservoirs: pathogenesis and obstacles to viral eradication and cure. AIDS. 2012;26(10):1261-1268.

3. Siliciano JD, Siliciano RF. Recent developments in the effort to cure HIV infection: going beyond $\mathrm{N}=1$. J Clin Invest. 2016;126(2):409-414.

4. Lederman MM, Funderburg NT, Sekaly RP, Klatt NR, Hunt PW. Residual immune dysregulation syndrome in treated HIV infection. Adv Immunol. 2013;119:51-83.

5. Heaton RK, et al. HIV-associated neurocognitive disorders persist in the era of potent antiretroviral therapy: CHARTER Study. Neurology. 2010;75(23):2087-2096.

6. McGuire JL, Barrett JS, Vezina HE, Spitsin S, Douglas SD. Adjuvant therapies for HIV-associated neurocognitive disorders. Ann Clin Transl Neurol. 2014;1(11):938-952.

7. Gelman BB. Neuropathology of HAND with suppressive antiretroviral therapy: encephalitis and neurodegeneration reconsidered. Curr HIV/AIDS Rep. 2015;12(2):272-279.

8. d'Ettorre G, et al. What happens to cardiovascular system behind the undetectable level of HIV viremia? AIDS Res Ther. 2016;13:21.

9. Douglas SD, Leeman SE. Neurokinin-1 receptor: functional significance in the immune system in reference to selected infections and inflammation. Ann N Y Acad Sci. 2011;1217:83-95.

10. Douglas SD, Lai JP, Tuluc F, Schwartz L, Kilpatrick LE. Neurokinin-1 receptor expression and function in human macrophages and brain: perspective on the role in HIV neuropathogenesis. Ann N Y Acad Sci. 2008;1144:90-96.

11. Ho WZ, Douglas SD. Substance P and neurokinin-1 receptor modulation of HIV. J Neuroimmunol. 2004;157(1-2):48-55.

12. Li Y, Douglas SD, Song L, Sun S, Ho WZ. Substance P enhances HIV-1 replication in latently infected human immune cells. $J$ Neuroimmunol. 2001;121(1-2):67-75.

13. Tuluc F, Lai JP, Kilpatrick LE, Evans DL, Douglas SD. Neurokinin 1 receptor isoforms and the control of innate immunity. Trends Immunol. 2009;30(6):271-276.

14. Douglas SD, et al. Elevated substance P levels in HIV-infected men. AIDS. 2001;15(15):2043-2045.

15. Douglas SD, et al. Elevated substance P levels in HIV-infected women in comparison to HIV-negative women. AIDS Res Hum Retroviruses. 2008;24(3):375-378.

16. Bost KL. Tachykinin-modulated anti-viral responses. Front Biosci. 2004;9:1994-1998.

17. Ho WZ, et al. Substance P modulates human immunodeficiency virus replication in human peripheral blood monocyte-derived macrophages. AIDS Res Hum Retroviruses. 1996;12(3):195-198.

18. Lai JP, Ho WZ, Zhan GX, Yi Y, Collman RG, Douglas SD. Substance P antagonist (CP-96,345) inhibits HIV-1 replication in human mononuclear phagocytes. Proc Natl Acad Sci U S A. 2001;98(7):3970-3975.

19. Wang X, Douglas SD, Lai JP, Tuluc F, Tebas P, Ho WZ. Neurokinin-1 receptor antagonist (aprepitant) inhibits drug-resistant HIV-1 infection of macrophages in vitro. J Neuroimmune Pharmacol. 2007;2(1):42-48.

20. Manak MM, et al. Anti-HIV-1 activity of the neurokinin-1 receptor antagonist aprepitant and synergistic interactions with other antiretrovirals. AIDS. 2010;24(18):2789-2796.

21. Tebas $\mathrm{P}$, et al. A randomized, placebo controlled, double masked phase IB study evaluating the safety and antiviral activity of aprepitant, a neurokinin-1 receptor antagonist in HIV-1 infected adults. PLoS One. 2011;6(9):e24180.

22. Tebas $\mathrm{P}$, et al. Reduction of soluble CD163, substance $\mathrm{P}$, programmed death 1 and inflammatory markers: phase $1 \mathrm{~B}$ trial of aprepitant in HIV-1-infected adults. AIDS. 2015;29(8):931-939.

23. Hargreaves R. Imaging substance P receptors (NK1) in the living human brain using positron emission tomography. J Clin Psychiatry. 2002;63(suppl 11):18-24.

24. Monaco-Shawver L, et al. Substance P inhibits natural killer cell cytotoxicity through the neurokinin-1 receptor. J Leukoc Biol. 2011;89(1):113-125.

25. Kramer MS, et al. Distinct mechanism for antidepressant activity by blockade of central substance P receptors. Science. 1998;281(5383):1640-1645.

26. Ratti E, et al. Full central neurokinin-1 receptor blockade is required for efficacy in depression: evidence from orvepitant clinical studies. J Psychopharmacol (Oxford). 2013;27(5):424-434.

27. Ratti E, et al. Efficacy of vestipitant, a neurokinin-1 receptor antagonist, in primary insomnia. Sleep. 2013;36(12):1823-1830.

28. Steinhoff MS, von Mentzer B, Geppetti P, Pothoulakis C, Bunnett NW. Tachykinins and their receptors: contributions to physi- 
ological control and the mechanisms of disease. Physiol Rev. 2014;94(1):265-301.

29. Highlights of prescribing information Emend. Merck. https://www.merck.com/product/usa/pi_circulars/e/emend/emend_ pi.pdf. Accessed September 26, 2017.

30. Bergström M, et al. Human positron emission tomography studies of brain neurokinin 1 receptor occupancy by aprepitant. Biol Psychiatry. 2004;55(10):1007-1012.

31. Barrett JS, McGuire J, Vezina H, Spitsin S, Douglas SD. PET measurement of receptor occupancy as a tool to guide dose selection in neuropharmacology: are we asking the right questions? J Clin Psychopharmacol. 2013;33(6):725-728.

32. Rupniak NMJ, Kramer MS. NK1 receptor antagonists for depression: Why a validated concept was abandoned. $J$ Affect Disord. 2017;223:121-125.

33. Ratti E, et al. Results from 2 randomized, double-blind, placebo-controlled studies of the novel NK1 receptor antagonist casopitant in patients with major depressive disorder. J Clin Psychopharmacol. 2011;31(6):727-733.

34. Barrett JS, et al. Pharmacologic rationale for the NK1R antagonist, aprepitant as adjunctive therapy in HIV. J Transl Med. 2016;14(1):148.

35. Barrett JS, et al. Modeling and simulation approach to support dosing and study design requirements for treating HIV-related neuropsychiatric disease with the NK1-R antagonist aprepitant. Curr HIV Res. 2014;12(2):121-131.

36. Gold L, et al. Aptamer-based multiplexed proteomic technology for biomarker discovery. PLoS One. 2010;5(12):e15004.

37. Gold L, Walker JJ, Wilcox SK, Williams S. Advances in human proteomics at high scale with the SOMAscan proteomics platform. N Biotechnol. 2012;29(5):543-549.

38. Huskey SE, et al. The metabolic disposition of aprepitant, a substance P receptor antagonist, in rats and dogs. Drug Metab Dispos. 2004;32(2):246-258.

39. Merck. Drug approval package: EMEND (Aprepitant) NDA \#21-549. 2003. https://www.accessdata.fda.gov/drugsatfda_docs/ nda/2003/21-549_Emend.cfm. Accessed September 7, 2017.

40. Rameshwar P, et al. The dynamics of bone marrow stromal cells in the proliferation of multipotent hematopoietic progenitors by substance P: an understanding of the effects of a neurotransmitter on the differentiating hematopoietic stem cell. J Neuroimmunol. 2001;121(1-2):22-31.

41. Giannarelli C, Klein RS, Badimon JJ. Cardiovascular implications of HIV-induced dyslipidemia. Atherosclerosis. 2011;219(2):384-389.

42. Patro-Malysza J, et al. The impact of substance $\mathrm{P}$ on the pathogenesis of insulin resistance leading to gestational diabetes. Curr Pharm Biotechnol. 2014;15(1):32-37.

43. Karagiannides I, et al. Substance P (SP)-neurokinin-1 receptor (NK-1R) alters adipose tissue responses to high-fat diet and insulin action. Endocrinology. 2011;152(6):2197-2205.

44. Ramalho R, et al. Substance P antagonist improves both obesity and asthma in a mouse model. Allergy. 2013;68(1):48-54.

45. Schaab M, et al. Novel regulatory mechanisms for generation of the soluble leptin receptor: implications for leptin action. PLoS One. 2012;7(4):e34787.

46. Drug Record: Aprepitant. NIDDK Website. https://livertox.nlm.nih.gov/Aprepitant.htm. Accessed September 7, 2017.

47. Spitsin S, Meshki J, Winters A, Tuluc F, Benton TD, Douglas SD. Substance P-mediated chemokine production promotes monocyte migration. J Leukoc Biol. 2017;101(4):967-973.

48. Farber DL, Yudanin NA, Restifo NP. Human memory T cells: generation, compartmentalization and homeostasis. Nat Rev Immunol. 2014;14(1):24-35.

49. Azma T, Tuluc F, Ito T, Aoyama-Mani C, Kawahito S, Kinoshita H. Mechanisms of action of anesthetics for the modulation of perioperative thrombosis: evidence for immune mechanisms from basic and clinical studies. Curr Pharm Des. 2014;20(36):5779-5793.

50. Dragoş D, Tănăsescu MD. The effect of stress on the defense systems. J Med Life. 2010;3(1):10-18.

51. Muñoz M, Coveñas R. NK-1 receptor antagonists: a new paradigm in pharmacological therapy. Curr Med Chem. 2011;18(12):1820-1831.

52. Johnson MB, Young AD, Marriott I. The Therapeutic Potential of Targeting Substance P/NK-1R Interactions in Inflammatory CNS Disorders. Front Cell Neurosci. 2016;10:296.

53. Mashaghi A, Marmalidou A, Tehrani M, Grace PM, Pothoulakis C, Dana R. Neuropeptide substance P and the immune response. Cell Mol Life Sci. 2016;73(22):4249-4264.

54. Porcheray F, Samah B, Léone C, Dereuddre-Bosquet N, Gras G. Macrophage activation and human immunodeficiency virus infection: HIV replication directs macrophages towards a pro-inflammatory phenotype while previous activation modulates macrophage susceptibility to infection and viral production. Virology. 2006;349(1):112-120.

55. Itoh N. FGF10: A multifunctional mesenchymal-epithelial signaling growth factor in development, health, and disease. Cytokine Growth Factor Rev. 2016;28:63-69.

56. Krueger $\mathrm{S}$, et al. Induction of premalignant host responses by cathepsin $\mathrm{x} / \mathrm{z}$-deficiency in Helicobacter pylori-infected mice. PLoS One. 2013;8(7):e70242

57. Wu D, Paul DJ, Zhao X, Douglas SD, Barrett JS. A sensitive and rapid liquid chromatography-tandem mass spectrometry method for the quantification of the novel neurokinin-1 receptor antagonist aprepitant in rhesus macaque plasma, and cerebral spinal fluid, and human plasma with application in translational NeuroAIDs research. J Pharm Biomed Anal. 2009;49(3):739-745.

58. Bates D, Mächler M, Bolker B, Walker S. Fitting linear mixed-effects models using 1me4. J Stat Softw. 2015;67(1):1-48

59. R Team Core. R: A language and environment for statistical computing. R Foundation for Statistical Computing, Vienna, Austria.

60. Foxe J, Weisberg S. An R Companion to Applied Regression. 2nd ed. London, United Kingdom: Sage Publications; 2011.

61. Wu G, Dawson E, Duong A, Haw R, Stein L. ReactomeFIViz: a Cytoscape app for pathway and network-based data analysis. F1000Res. 2014;3:146. 